\title{
Assistência domiciliária em saúde: um olhar crítico sobre a produção científica de enfermagem
}

\author{
Home healthcare: a critical appraisal on nursing sicientific production \\ Atención domiciliária de salud: una crítica sobre la producción científica de enfermería
}

\author{
Ana Larissa Gomes Machado', Maria Josefina da Silva", Consuelo Helena Aires Freitas ${ }^{1}$ \\ 'Universidade Estadual do Ceará. Graduação em Enfermagem. Fortaleza, CE \\ "Universidade Federal do Ceará. Departamento de Enfermagem. Fortaleza, CE
}

Submissão: 24/08/2009

Aprovação: 20/11/2010

\section{RESUMO}

Objetivou-se analisar a produção científica da enfermagem acerca da assistência domiciliar em saúde, a partir de levantamento bibliográfico em endereços eletrônicos de periódicos de enfermagem, relativos ao período de 2000 a 2007. Identificaram-se duas temáticas: organização da assistência domiciliária e percepção dos cuidadores e de suas famílias acerca do cuidado domiciliário. Observou-se o interesse dos autores em definir conceitos Que traduzem as formas de organização da assistência profissional no domicílio e em identificar as características dos cuidadores e de seus familiares. Em conclusão, a enfermagem tem inserido o cuidado no domićlio como objeto de suas pesquisas, mas ainda de forma incipiente, fato Que reforça a necessidade de ampliação e divulgação das investigações da profissão.

Descritores: Serviços de assistência domiciliar; Cuidados de enfermagem; Cuidados domiciliares de saúde.

\section{ABSTRACT}

The aim was to analyze the nursing scientific production concerning to home health care, from bibliography in electronic addresses of nursing journals, covering the period between 2000 and 2007. Two themes were identified: organization of home care and perceptions of caregivers and their families about the home care. There was the interest of authors in define concepts that reflect the ways of organizing the professional assistance in home and in identify the characteristics of caregivers and their families. In conclusion, the nursing has entered the care at home as the object of their search, but still so low, a fact which reinforces the need for expansion and dissemination of research of the profession.

Key words: Home care services; Nursing care; Home nursing.

\section{RESUMEN}

El objetivo fue analizar la producción científica de enfermería respecto a la asistencia domiciliaria en salud, a partir del levantamiento bibliográfico en direcciones electrónicas de periódicos de enfermería, relativo al período de 2000 a 2007. Se identificaron dos temáticas: organización de la asistencia domiciliaria y percepción de los cuidadores y de sus familias sobre el cuidado domiciliario. Se observó el interés de los autores en definir conceptos Que traducen las formas de organización de la asistencia profesional en el domicilio y en identificar las características de los cuidadores y de sus familiares. En conclusión, la enfermería ha inserido el cuidado en el domicilio como objeto de sus investigaciones, pero todavía de forma incipiente, hecho que refuerza la necesidad de ampliación y divulgación de las investigaciones de la profesión.

Descriptores: Servicios de atención de salud a domicilio; Atención de enfermería; Atención domiciliaria de salud.

AUTOR CORRESPONDENTE Ana Larissa Gomes Machado. Universidade Estadual do Ceará. Campus do Itaperi. Avenida Paaranjana, 1700. Fortaleza, CE. E-mail: analarissag@hotmail.com 


\section{INTRODUÇÃO}

A proximidade com o sujeito doente tem sido buscada em vários setores da assistência à saúde. Em virtude das características singulares que detém, a assistência domiciliária (AD) destaca-se como modalidade de cuidado Que pressupõe competências profissionais ampliadas direcionadas ao sujeito, família e comunidade.

A AD acontece em cenários e contextos peculiares. Está presente nos três níveis de atenção à saúde e envolve equipe multiprofissional. Apesar da ampliação do seu objeto de trabalho, a AD ainda é realizada tendo como referência o modelo clínico-hospi$\operatorname{talar}^{(1)}$.

Esse estudo é parte de um trabalho mais extenso, no Qual foi desenvolvido processo investigatório em contexto domiciliar acerca da vivência de cuidadores familiares de vítimas de acidente cerebrovascular $(\mathrm{AVC})^{(2)}$.

Inicialmente realizou-se um estudo bibliográfico em periódicos, Quando foi analisada a produção científica da enfermagem sobre o cuidado realizado no domicílio.

Para o profissional atuar na AD é necessário o entendimento acerca do significado Que a presença do familiar doente no domicílio tem para a família ${ }^{(2)}$. As pesquisas sobre o assunto exploram conceitos importantes nesse cenário, como cuidador familiar, domicílio, visita domiciliar, rede de apoio social da família e as definições acerca da expressão "assistência domiciliária".

A produção do conhecimento sobre a temática encontra-se em processo de emergência, em virtude de sua magnitude e do aumento da demanda social pela $\mathrm{AD}$ no cenário de saúde brasileiro.

Por se tratar de assunto ainda pouco discutido, do ponto de vista da investigação científica, é Que se objetivou analisar a produção científica da enfermagem acerca da assistência domiciliar em saúde. Nesse artigo, está sendo apresentado o conhecimento relacionado à organização da $\mathrm{AD}$ em alguns serviços de saúde e à percepção dos cuidadores e de suas famílias acerca do cuidado domiciliário, Que se constituíram nas temáticas relevantes a partir dos artigos analisados.

\section{MÉTODO}

Essa pesQuisa constituiu-se num recorte, reunindo 20 artigos, a partir de pesquisa bibliográfica com 30 artigos, localizados nos endereços eletrônicos de periódicos de enfermagem correspondentes ao período de 2000-2007. Foram excluídos do estudo 10 artigos, os Quais foram publicados fora do período de interesse e não estavam disponíveis na íntegra em versão eletrônica.

$\mathrm{O}$ acesso às fontes de pesquisa ocorreu no período de julho a dezembro de 2007 . A coleta dos dados deu-se com a consulta sistemática, via internet, aos endereços eletrônicos dos periódicos, a partir dos descritores: "serviços de assistência domiciliar"; "cuidados de enfermagem" e "cuidados domiciliares de saúde", obedecendo-se aos seguintes critérios de inclusão: acessibilidade, disponibilidade dos números na íntegra e relação com a temática da assistência de enfermagem domiciliária.

Para manter o anonimato das publicações, os periódicos Que atendiam aos critérios de seleção propostos forma identificados como Periódico 1, Periódico 2, Periódico 3 e Periódico 4.

A análise dos dados tomou como referencial teórico a análise de conteúdo de Bardin que envolveu as seguintes etapas: a pré- análise, a análise e o tratamento dos resultados e interpretação ${ }^{(3)}$.

A pré-análise compreendeu a organização, a leitura flutuante e a preparação do material a ser analisado durante a peseuisa, Quando se verificou se os trabalhos realmente tratavam do tema abordado. A análise correspondeu ao período de exploração do material. Aqui se concentraram as fases de recorte do corpus, categorização e descrição das categorias. Na fase de tratamento dos resultados e de interpretação trabalhou-se na linha Quati-Qualitativa, por meio da identificação e discussão das seguintes variáveis: titulação dos autores, procedência dos autores por região, natureza ou abordagem dos estudos e o conteúdo dos artigos Quanto ao conhecimento dos sujeitos sobre assistência domiciliária e a organização dessa modalidade de cuidado em diferentes serviços.

Apresenta-se a produção do conhecimento a partir das temáticas "organização da assistência domiciliária" e "percepção dos cuidadores/familiares acerca do cuidado domiciliário". A primeira reúne as seguintes categorias: 1 - análise de serviços de saúde Que oferecem $A D, 2$ - instrumentos para a $A D$, congregando as subcategorias: visita domiciliária, instrumento de abordagem psicossocial à família e aplicação de modelos assistenciais em AD; 3 classificação de pacientes em AD.

A segunda temática reúne a categoria: 1 - vivência do cuidador familiar, Que congrega as subcategorias: caracterização do cuidador e significado das ações do profissional e do cuidado domiciliar para o cuidador.

\section{RESULTADOS}

A análise dos artigos resultou na identificação das características dos estudos e de seus autores, como pode ser observado na Tabela 1. Destacam-se os anos de 2004 e 2005 Que apresentaram maior produção acerca da temática estudada, com 6(30\%) e 9(45\%) publicações, respectivamente. As titulações mais recorrentes entre os autores dos artigos analisados foram doutorado, 7(35\%), e mestrado, 4(20\%).

Acerca da região do País onde foram publicados os artigos, a região Sudeste, representada nesse trabalho por São Paulo, Minas Gerais e Rio de Janeiro, respondeu por $80 \%$ das publicações, em contrapartida, apenas 5\% estavam na região Nordeste, e 15\% na região Sul.

Em relação à abordagem dada pelos autores nos artigos analisados, a pesquisa Qualitativa foi mais utilizada, correspondendo a 13(65\%) publicações. A abordagem Quanti/Quali foi utilizada em apenas 2(10\%) dos estudos analisados.

\section{DISCUSSÃO}

Foram considerados trabalhos de mestrado e/ou doutorado aqueles cujos autores identificaram-se como mestrandos e doutorandos ou Que estavam publicando recortes de suas dissertações e/ou teses. Como trabalhos de iniciação científica os Que possuíam alunos de graduação em enfermagem como autores, integrantes de algum projeto de pesquisa e docentes aqueles cujos autores identificaram-se como professores de graduação/pós-graduação de alguma instituição de ensino superior.

Apesar do crescente interesse de profissionais da saúde e de pesQuisadores por compreender as especificidades da atenção do- 
Tabela 1. Variáveis analisadas.

\begin{tabular}{lcc}
\hline Variáveis & n & \% \\
\hline Periódico & & \\
P1 & 5 & 25 \\
P2 & 6 & 30 \\
P3 & 5 & 25 \\
P4 & 4 & 20 \\
Ano de Publicação & & \\
2000 & - & - \\
2001 & - & - \\
2002 & 2 & 10 \\
2003 & 1 & 5 \\
2004 & 6 & 30 \\
2005 & 9 & 45 \\
2006 & 2 & 10 \\
Titulação dos autores & & \\
Doutorado & 7 & 35 \\
Mestrado & 4 & 20 \\
Especialização & 1 & 5 \\
Docente & 7 & 35 \\
Iniciação Científica & 1 & 5 \\
Procedência dos autores por região & & \\
Nordeste & 1 & 5 \\
Sul & 3 & 15 \\
Sudeste & 16 & 80 \\
Natureza ou abordagem dos estudos & & \\
Quantitativa & 5 & 25 \\
Qualitativa & 13 & 65 \\
Quanti/Qualitativa & 2 & 10 \\
\hline
\end{tabular}

miciliária em saúde, a produção científica ainda se apresenta de forma incipiente, com um número peQueno de artigos nos periódicos analisados.

Um aspecto importante a ser ressaltado é o Quantitativo de pesquisas oriundas de cursos de doutorado e de docentes. Sabese Que as agências de fomento à pesquisa exigem a pu-blicação das construções científicas realizadas na academia. Isso implica diretamente no estímulo aos doutorandos, mestrandos e docentes para Que publieuem suas investigações.

Os trabalhos de iniciação científica e de especialização não se apresentaram com números representativos nas pesQuisas, porém representam uma importante parcela de pesquisadores em preparação para ingressar em cursos de pós-graduação stricto sensu e na docência do ensino superior, momentos de formação nos Quais desenvolverão além de outras habilidades, a consciência e o interesse em publicar seus estudos.

A performance dos estados do sudeste é o resultado do ambiente de cooperação entre instituições e políticas públicas, o Que possibilita a criação de cursos de mestrado e doutorado na região. Além disso, os periódicos com maior número de artigos são órgãos oficiais de divulgação científica das próprias instituições de ensino superior nas Quais os autores cursam graduação/pós-graduação ou lecionam.

Outro aspecto importante a ser discutido é a natureza das pesQuisas analisadas. A abordagem Qualitativa respondeu por $65 \%$ dos artigos, apesar da simpatia das agências de fomento à pesQuisa pela abordagem Quantitativa. Os enfermeiros, seja pela proximidade de seu objeto de estudo ou de trabalho, realizam peseui- sas Qualitativas para explorar diversas temáticas e nesse estudo analisam a assistência domiciliar sob o ponto de vista dos trabalhadores e da clientela Que recebe os cuidados.

Tecidas as considerações sobre as características dos estudos e de seus autores, segundo aspectos Quantitativos, essa pesquisa destaca as temáticas recorrentes nos artigos analisados acerca da atenção domiciliar. Inicialmente será analisada, a partir dos resultados apresentados nos estudos, a organização dos serviços públicos e privados Que oferecem assistência à saúde no domicílio e, em seguida, a percepção dos cuidadores/familiares acerca do cuidado domiciliário.

\section{A organização da assistência domiciliária}

Essa temática reúne a produção do conhecimento relacionada à operacionalização dos serviços de assistência domiciliária. A leitura dos trabalhos revelou a preocupação dos pesquisadores em: 1expor as experiências de alguns serviços de saúde, tomando algum indicador para avaliar o cuidado prestado; 2- avaliar os instrumentos utilizados para cuidar no domicílio, como a visita domiciliária e a aplicação de modelos assistenciais; 3- conhecer as reais demandas de cuidado dos pacientes Que necessitam de assistência em suas residências.

As pesquisas trazem de forma sistemática conceitos Que traduzem as formas de organização da assistência profissional no domicílio. Destacam-se os conceitos de visita domiciliária, entendida como atendimento realizado por profissional e/ou equipe de saúde na residência do cliente, com o objetivo de avaliar suas necessidades, de seus familiares e do ambiente para construir um plano de cuidados $^{(4)}$, atendimento domiciliar, o Qual compreende as atividades assistenciais exercidas pelo profissional na residência do cliente e internação domiciliar, entendida como atividades especializadas, com a oferta de recursos humanos, materiais e medicamentos, assemelhando-se ao cuidado oferecido no ambiente hospitalar ${ }^{(5)}$.

A partir do entendimento desses conceitos, as narrativas acerca das experiências de alguns serviços de assistência domiciliária em saúde estabelecem como critérios de avaliação os custos com recursos materiais, como dietas e medicamentos ${ }^{(6)}$ e as formas como os serviços públicos ou privados se organizam para instituir a mudança no modelo de atenção à saúde, acreditando-se Que a assistência domiciliária possui potência para essa transformação(7).

Tecendo considerações acerca da avaliação da assistência domiciliária, observa-se que as pesquisas elegeram a visita domiciliária como importante estratégia assistencial, pois permite a observação do contexto familiar e a disponibilidade de recursos na comunidade ${ }^{5}$. Aliam-se à visita, a aplicação de modelos assistenciais e de instrumentos de abordagem à família, como formas de apreender as expressões de vida e de saúde/doença apresentadas pelo indivíduo e família, em virtude do vazio teórico-instrumental existente ${ }^{(1,8)}$.

A apreensão das expressões de vida dos cuidadores domiciliares e de seus familiares torna-se uma difícil tarefa para os profissionais de saúde, Quando a formação acadêmica é limitante, ou seja, não consegue ampliar a abordagem do sujeito para além da dimensão clínica tradicional( ${ }^{(9)}$.

Essa dimensão consiste no fazer profissional voltado para a doença, pautado em saberes e técnicas Que não valorizam o sujeito como ser capaz de adeuirir conhecimentos para cuidar de si e interferir na evolução do seu processo saúde/doença. 
A assistência domiciliária para ser efetiva necessita de profissionais com uma visão ampliada de clínica, a Qual volte seu olhar para o ser humano e suas subjetividades. O envolvimento da família no tratamento dos pacientes no domicílio remete à necessidade da construção de novas formas de cuidado em saúde ${ }^{(1)}$.

Para Que o cuidado no domicílio seja efetivo, contudo, também é preciso atenção ao dimensionamento de recursos humanos. Nessas circunstâncias, destacam-se os profissionais de enfermagem na implementação da assistência domiciliar e na manutenção da sua Qualidade $^{(10)}$.

O trabalho do enfermeiro no contexto domiciliar consiste em cuidar, ajudar a cuidar, orientar e encaminhar. Os seus conhecimentos e o vivenciar das situações possibilitam-lhe saber como agir. Não há como prever uma rotina de atendimento pela dinâmica de cada domicílio. Exigem-se do profissional capacidade de readaptação constante, criatividade e atitude interdisciplinar ${ }^{(11-12)}$.

Considerando-se esses aspectos, é importante que o enfermeiro conheça as reais necessidades de cuidado de seus clientes, a partir da determinação do grau de dependência em relação à equipe de enfermagem $^{(13)}$. O desempenho da equipe de enfermagem relaciona-se diretamente à adeQuação do tempo de permanência do profissional no domicílio, de acordo com as necessidades do paciente/família.

\section{Percepção dos cuidadores/familiares acerca do cuidado domi- ciliário}

A construção dessa categoria ocorreu a partir da observação do interesse dos autores dos estudos analisados em investigar o significado Que o cuidado domiciliário tem para os cuidadores, as pessoas cuidadas e as famílias.

A análise dos trabalhos resultou na identificação dos principais objetivos dos autores: 1- identificar as características dos cuidadores e dos familiares por eles cuidados e 2- conhecer os sentimentos e as percepções dos cuidadores e familiares acerca da assistência domiciliária e das ações dos profissionais no domicílio.

No tocante à caracterização dos cuidadores, os seguintes aspectos foram explorados por Quatro estudos: sexo, idade, estado civil, renda, escolaridade, religião, parentesco com a pessoa cuidada, tempo de cuidado, tipo de cuidado realizado e formação técnica ${ }^{(1-3,6)}$. Quanto às características dos familiares sob cuidados no domicílio, foram explorados em Quatro artigos os seguintes aspectos: sexo, escolaridade, renda e o tipo de doença da Qual são portadores ${ }^{(1,6-8)}$.

É evidente a presença feminina assumindo o papel de cuidadora, com idades compreendidas entre 30 e 82 anos, pertencentes ao grupo familiar do ser cuidado, principalmente filhas e esposas, com poucos anos de estudo e sem formação específica para o cuidado domiciliar.

A respeito dos familiares cuidados no domicílio, prevalecem nos estudos aqueles com idade superior a 61 anos, com manifestações de complicações de doenças cardiovasculares e neoplasias, e aqueles sem possibilidades terapêuticas de cura.

A partir da constatação da importância e da presença da família no cuidado à crescente população de pessoas dependentes de cuidados no domicílio, alguns autores interessaram-se por conhecer os significados e os sentimentos dos cuidadores e familiares acerca do cuidado domiciliar ${ }^{(1-5,7,9)}$.

Apenas dois artigos não apresentaram resultados para esse aspecto da temática ${ }^{(6,8)}$. Os demais abordaram, dentre outras Questões não menos relevantes, sentimentos dos cuidadores sobre o surgimento de fenômenos como a dependência e o apego do familiar com o advento da doença, a necessária reorganização familiar para oferecer cuidado efetivo no domicílio, o significado da assistência domiciliária prestada pelos profissionais, a percepção de sua condição de cuidador (a), as motivações para cuidar, a sobrecarga física e emocional, o cuidado em tempo integral, algumas vezes com o apoio de amigos, vizinhos e da família e, outras vezes, o cuidado solitário.

Constatou-se, portanto, Que os autores voltaram seus olhares para os sentimentos e significados do cuidador domiciliar por entendê-lo como ator social Que presta incomensurável serviço à família, à sociedade e ao sistema de saúde brasileiro.

As respostas do sistema de saúde e de políticas públicas voltadas ao cuidado dos idosos e de pessoas dependentes ainda são insuficientes no País, o Que constitui o desafio de buscar novas estratégias assistenciais Que atendam essa população. O cuidado no domicílio ainda tem caráter informal, sendo o cuidador familiar a pessoa fundamental para sua existência. A organização da assistência domiciliar exige a definição de políticas assistenciais Que envolvam disponibilidade de tecnologias e de capacitação de cuidadores.

Para os cuidadores dos estudos analisados, o cuidado domiciliário é considerado uma tarefa difícil, em virtude das mudanças Que impõe a suas vidas, do despertar de sentimentos como a obrigação de cuidar dos pais e/ou do marido, da exacerbação dos conflitos entre os familiares e do aprendizado necessário para cui$\operatorname{dar}^{(14-16)}$

Em contrapartida, familiares e cuidadores apontaram nas pesQuisas aspectos positivos resultantes da interação com os profissionais nos domicílios. Dentre estes, a visualização do trabalho da enfermeira, a garantia de tranquilidade e segurança com a presença da enfermeira, ser auxiliado em momentos de urgência e ser ensinado a cuidar ${ }^{(11,17)}$.

Além dos aspectos subjetivos elencados acima, os autores também se preocuparam em respaldar seus estudos ao definir conceitos relacionados à temática, a exemplo de "cuidador informal e formal", Que estavam presentes nas pesquisas Que objetivavam compreender os sentimentos dos cuidadores acerca desses conceitos.

O primeiro foi conceituado como um membro do grupo familiar ou amigo e vizinho residentes no local onde o cuidado é prestado, sem receber nenhuma remuneração pela ajuda oferecida e Que não tem uma formação específica. A atenção informal emerge das relações interpessoais e se constrói no cotidiano familiar e social ${ }^{(18-19)}$.

lá o cuidador formal foi definido como pessoa com formação específica na área da saúde Que possui vínculo empregatício ou alguma forma de remuneração para exercer ações de cuidado no domicílio ${ }^{(19)}$.

Na pesquisa que abordou o cuidado familiar a pessoas dependentes sem possibilidade terapêutica de cura, os conceitos de terapia paliativa e de cuidados paliativos também foram identificados ${ }^{(14)}$.

\section{CONSIDERAÇÕES FINAIS}

A busca da enfermagem por seu próprio corpo de conhecimentos avançou muito ao longo do tempo, voltando sua atenção para áreas diversas como a assistência, o ensino e a pesquisa. Em vir- 
tude disso, é importante a análise da produção científica de enfermeiros para ampliação e maior divulgação do conhecimento da enfermagem.

O presente estudo proporcionou a reflexão sobre a assistência domiciliária em saúde e destacou a enfermagem como profissão essencialmente presente nesse contexto.

Infere-se, da pesquisa realizada, Que a assistência domiciliária está em ascensão e, uma vez realizada com responsabilidade e competência, traz muitos benefícios para as pessoas e suas famílias. Ainda são incipientes as pesquisas e os instrumentos de avaliação dessa modalidade de cuidado, fato Que favorece os estudos acerca da temática, já Que a mesma se faz presente em serviços de saúde públicos e privados e exige dedicação do profissional.

Entende-se Que a enfermagem deve ampliar suas investigações acerca do cuidado domiciliário, e assim, contribuir para a divulgação do conhecimento da profissão e para o reconhecimento da família como parceira no cuidado, uma vez Que as atividades são compartilhadas com os membros familiares dos pacientes, os Quais necessitam de escuta e capacitação para oferecer um cuidado humano e de Qualidade.

\section{REFERÊNCIAS}

I. Püschel VAA, Ide CAC, Chaves EC. Instrumento para a abordagem psicossocial do indivíduo e da família na assistência domiciliar - condições de aplicabilidade. Acta Paul Enferm 2005; 18(2): 203-12.

2. Machado ALG. A vivência de cuidadores familiares de pessoa incapacitada por acidente vascular cerebral: uma abordagem interacionista [dissertação]. Fortaleza: Universidade Estadual do Ceará; 2008.

3. Bardin L. Análise de conteúdo. Lisboa: Edições 70; 1977.

4. Fabrício SCC, Webbe G, Nassur FB, Andrade II. Assistência domiciliar: a experiência de um hospital privado do interior paulista. Rev Latino-am Enfermagem 2004; I 2(5): 72 I-6.

5. Roese A, Lopes MIM. A visita domiciliar como instrumento de coleta de dados de pesquisa e vigilância em saúde: relato de experiência. Rev Gaúcha Enferm 2004; 25(1): 98-1 II .

6. Mesquita SRAM, Anselmi ML, Santos CB, Hayashida M. Programa Interdisciplinar de internação domiciliar de Marília-SP: custos de recursos materiais consumidos. Rev Latino-am Enfermagem 2005; 13(4): 555-61.

7. Pereira MJB, Mishima SM, Fortuna CM, Matumoto S. A assistência domiciliar- conformando o modelo assitencial e compondo diferentes interesses/necessidades do setor saúde. Rev Latino-am Enfermagem 2005; 13(6): 1001-10.

8. Fialho AVM, Pagliuca LMF, Soares E. AdeQuação da teoria do déficit de autocuidado no cuidado domiciliar à luz do modelo de Barnum. Rev Latino-am Enfermagem 2002; 10(5): 71520.

9. Machado ALG, Jorge MSB, Freitas CHA. A vivência do cuidador familiar de vítima de acidente vascular encefálico: uma abordagem interacionista. Rev Bras Enferm 2009; 62(2): 246-5 I.

10. Dal Ben LW, Gaidzinski RR. Home Care. Planejamento e administração da equipe de enfermagem. São Paulo: Andreoli; 2007.

11. Lacerda MR, Oliniski SR. A família e a enfermeira no contexto domiciliar: dois lados de uma realidade. Texto Contexto Enferm 2003; 12(3): 307-13.

12. Paskulin LMG, Dias VRFG. Como é ser cuidado em casa: as percepções dos clientes. Rev Bras Enferm 2002; 55(2): 140-5.

13. Dal Ben LW, Gaidzinski RR. Sistema de classificação de pacientes em assistência domiciliária. Acta Paul Enferm 2006; 19(1): 100-8.

14. Marques SM, Ferraz AF. A vivência do cuidado domiciliar durante o processo de morrer: a perspectiva de familiares cuidadores. Rev Mineira Enferm 2004; 8(1): 165-252.

15. Freire GD, Campos DR, Boemer MR. Compreendendo o paciente gravemente enfermo e sua família na realidade domiciliar. Rev Gaúcha Enferm 2004; 25(3): 346-56.

16. Marzari F, Perlini NMOG. Cuidar no domicílio: percepção de cuidadores familiares na área rural. Rev Mineira Enferm 2005; 9(4): 322-28.

17. Paskulin LMG, Eidt OR, Morais EP, Jansen M, Schossler T, Gonçalves A. Clientes idosos submetidos a artroplastia total de Quadril primária (ATQP): o Que pensam a respeito do cuidado recebido no domićlio. Acta Paul Enferm 2004; 17(2): 2 I I-2 I .

18. Rodrigues MR, Almeida RT. Papel do responsável pelos cuidados à saúde do paciente no domicílio - um estudo de caso. Acta Paul Enferm 2005; 18(1): 20-4.

19. Gonçalves AM, Sena RR, Dias DG, Queiróz CM, Dittz E, Vivas KL, et.al. Cuidadora domiciliar: por Que cuido? Rev Mineira Enferm 2005; 9(4): 315-20. 